\title{
In-Situ Liquid Cell TEM Studies of Electrochemical reactions in Lithium-ion Batteries
}

\author{
$\underline{\text { Wei Zhang }}{ }^{1}$, Daan Hein Alsem ${ }^{2}$, Norman Salmon ${ }^{2}$ and Feng Wang ${ }^{1}$ \\ ${ }^{1}$ Sustainable Energy Technologies Department, Brookhaven National Lab, Upton, New York, United \\ States 11973 \\ ${ }^{2}$ Hummingbird Scientific, Lacey, WA 98516
}

Lithium-ion batteries (LIBs) are the preferred energy storage devices for portable electronics and electric vehicles due to the long cycle life and high energy density [1]. The development of new electrodes for next-generation batteries requires better understanding how electrodes function by realtime tracking the electrochemical reactions in individual particles and the collective behavior of particles assembled in an electrode. Various available characterization methods, such as in-situ X-ray techniques, are powerful for real time tracking charge/discharge dynamics of electrodes in liquid electrochemical system, but with limited spatial resolution. Recently the development of in-situ TEM techniques enables real time observation of structural and chemical evolutions of electrodes during battery operation at high spatial and temporal resolution [2]. However, due to the use of an open-cell configuration in these platforms, solid or ionic liquid electrolyte needs to be used for compatibility with high vacuum in the TEM column. In order to study the lithium reaction with electrodes in liquid electrolyte as generally used in LIBs, we developed a liquid electrochemical cell with thin membrane windows to seal the liquid electrolyte, allowing for both TEM and X-ray measurements with the sample in liquid environments. Here, we present our recent results from the development and application of the liquid electrochemical cells for in-situ TEM studies of lithium transport and reaction in individual particles upon lithiation and delithiation.

For electrochemistry experiments, the electrochemical capabilities were developed inside a liquid cell TEM holder (designed by Hummingbird Scientific)[3], as shown in Figure 1a and b. This holder uses micro-fluidic techniques and two micro-fabricated chips each having an electron transparent $\mathrm{SiN}$ viewing windows (Figure 1c), and uses a sealing mechanism in which the chips can be secured on the tip of the holder and sealed from vacuum in the TEM column by means of a mechanical sealing mechanism using miniature O-rings and a simple yet reliable clamping solution. The micro-fabricated chips possess three electrodes with different metal options with electrical biasing capabilities. The active electrode material can be attached to metal on-chip leads - those are only exposed to the electrolyte in the TEM viewing area of the cell.

By using the liquid cell we performed in-situ studies of electrochemical reactions in single particles of two model systems, $\mathrm{LiFePO}_{4}$ and $\mathrm{FeF}_{2}$. They are the most extensively studied cathodes due to their intriguing phase transition behaviors, which are still under intense debate [2, 4]. For the in-situ measurements, a standard liquid electrolyte (e.g. $1 \mathrm{M} \mathrm{LiPF}_{6}$ salt in ethylene carbonate and dimethyl carbonate) is sealed within the thin membranes ( $50 \mathrm{~nm}$ or below) of the top and the bottom chips. Figure 2 shows the typical morphology of $\mathrm{FeF}_{2}$ nanoparticles, with a plate-like shape of about a few micrometers long but only several tens of nanometers thick. Good contrast of nanoplates in the liquid electrolyte was obtained by bright-field TEM imaging. The orientation of those nanoplates in the liquid electrolyte was also determined by electron diffraction. They are mostly oriented along $\{001\}$ or $\{110\}$ directions, allowing us to study the orientation-dependent chemical and structural evolution in single- 
crystalline $\mathrm{FeF}_{2}$ particles during lithiation and delithiation, in a combination of TEM imaging, diffraction and valence electron energy-loss spectroscopy [5]. Results from in-situ TEM studies of the lithium transport and electrochemical dynamics, as well as the complex reactions at the electrode-electrolyte interfaces in individual $\mathrm{FeF}_{2}$ and $\mathrm{LiFePO}_{4}$ nanoparticles, will be discussed [6].
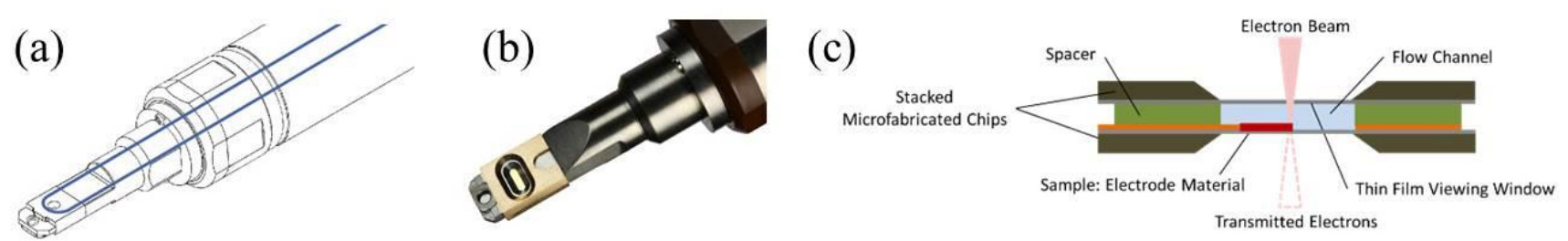

Figure 1. (a) Liquid flow path in liquid TEM flow holder, (b) in-situ TEM liquid holder tip, (c) crosssection of the liquid-electrochemical cell.
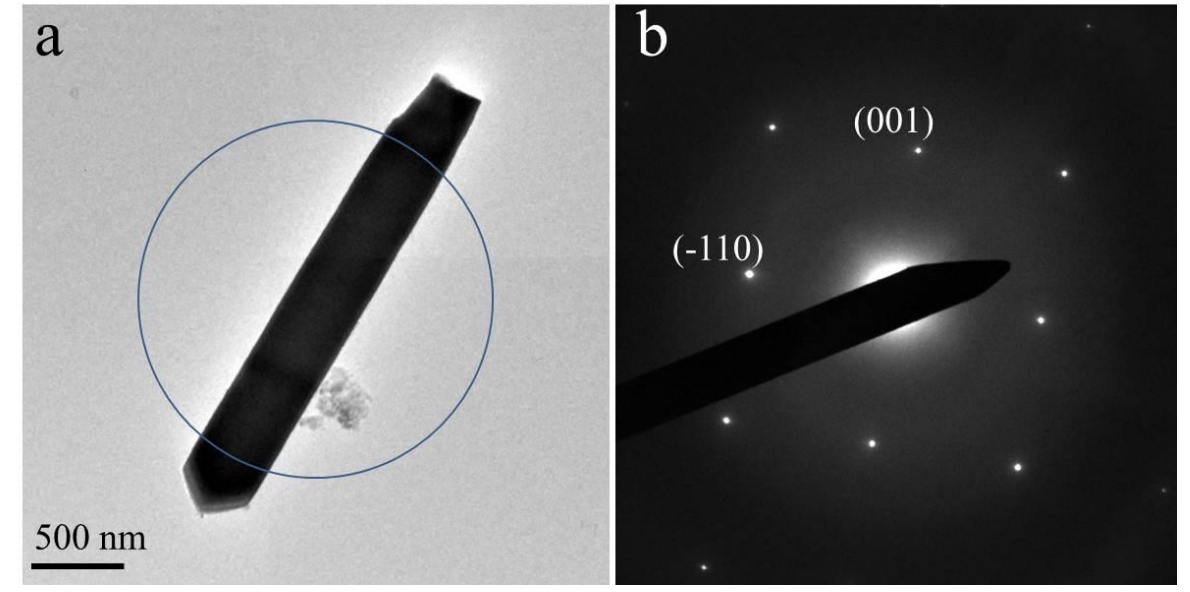

Figure 2. (a) Bright-field TEM image of a single $\mathrm{FeF}_{2}$ nanoplate in the presence of liquid electrolyte, (b) a diffraction pattern recorded from the region masked by blue circle of $\mathrm{FeF}_{2}$ nanoplate in (a), indicating the [110] zone axis.

\section{References:}

[1] Tarascon, J.-M.; Armand, M. Nature 414 (2001), 359-367.

[2] Wang, F.; Yu, H.-C.; Chen, M.-H.; Wu, L.; Pereira, N.; Thornton, K.; Van der Ven, A.; Zhu, Y.; Amatucci, G. G.; Graetz, J. Nature communications 3 (2012), 1201.

[3] Abellan, P.; Mehdi, B. L.; Parent, L. R.; Gu, M.; Park, C.; Xu, W.; Zhang, Y.; Arslan, I.; Zhang, J.G.; Wang, C.-M. Nano letters 14 (2014), 1293-1299.

[4] Malik, R.; Abdellahi, A.; Ceder, G. Journal of The Electrochemical Society 160 (2013), A3179A3197.

[5] Katherine, J.; Evans, J.E.; Aguiar, J.A.; Arslan, I; Browning, N.D. Microscopy and Microanalysis 18 (2012), 621.

[6] This work is financially supported by the Laboratory Directed Research and Development (LDRD) program at Brookhaven National Laboratory. Research carried out at the Center for Functional Nanomaterials, Brookhaven National Laboratory, is supported by the U.S. Department of Energy, Office of Basic Energy Sciences, under Contract No. DE-AC02-98CH10886. 